\title{
Transient Elastography for Significant Liver Fibrosis and Cirrhosis in Chronic Hepatitis B: A Meta-Analysis
}

\author{
Xiaolong Qi $\mathbb{D},{ }^{1,2}$ Min An, ${ }^{3}$ Tongwei Wu, ${ }^{2}$ Deke Jiang, ${ }^{2}$ Mengyun Peng, ${ }^{4}$ Weidong Wang $\mathbb{D},{ }^{5}$ \\ Jing Wang ${ }^{1},{ }^{4}$ Chunqing Zhang, ${ }^{1}$ and on behalf of the CHESS Study Group ${ }^{2}$ \\ ${ }^{1}$ Department of Gastroenterology, Shandong Provincial Hospital Affiliated to Shandong University, Jinan, China \\ ${ }^{2}$ CHESS, Hepatic Hemodynamic Lab, Institute of Hepatology, Nanfang Hospital, Southern Medical University, \\ Guangdong Provincial Research Center for Liver Fibrosis, Guangzhou, China \\ ${ }^{3}$ The Second School of Clinical Medicine, Southern Medical University, Guangzhou, China \\ ${ }^{4}$ Department of Hepatobiliary Disease, The Affiliated (T.C.M) Hospital of Southwest Medical University, Luzhou, China \\ ${ }^{5}$ Department of Hepatobiliary Surgery, Shunde Hospital, Southern Medical University, Foshan, China
}

Correspondence should be addressed to Weidong Wang; wangweidong1968@126.com and Jing Wang; lywj68@126.com

Received 9 December 2017; Accepted 25 February 2018; Published 24 May 2018

Academic Editor: Mingyu Sun

Copyright (C) 2018 Xiaolong Qi et al. This is an open access article distributed under the Creative Commons Attribution License, which permits unrestricted use, distribution, and reproduction in any medium, provided the original work is properly cited.

Background. The hepatitis B virus infection is a global health issue and the stage of liver fibrosis affects the prognosis in patients with chronic hepatitis $\mathrm{B}(\mathrm{CHB})$. We performed the meta-analysis describing diagnostic accuracy of transient elastography (TE) for predicting CHB-related fibrosis. Methods. We performed an adequate literature search to identify studies that assessed the diagnostic accuracy of TE in CHB patients using biopsy as reference standard. Hierarchical summary receiver-operating curves model and the bivariate mixed-effects binary regression model were applied to generate summary receiver-operating characteristic curves and pooled estimates of sensitivity and specificity. Results. The area under the summary receiver-operating curve for significant fibrosis and cirrhosis was 0.86 (95\% confidence interval (CI): 0.83-0.89) and 0.92 (95\% CI: 0.90-0.94), respectively. The sensitivity, specificity, and diagnostic odds ratio of TE for significant fibrosis were 0.78 (95\% CI: $0.73-0.81, p<0.01 ; I^{2}=85.59 \%$ ), 0.81 (95\% CI: $\left.0.77-0.84, p<0.01 ; I^{2}=88.20 \%\right)$, and 14.44 (95\% CI: $\left.10.80-19.31, p<0.01 ; I^{2}=100 \%\right)$ and for cirrhosis were 0.84 (95\% CI: $0.80-0.88, p<0.01 ; I^{2}=76.67 \%$ ), 0.87 (95\% CI: 0.84-0.90, $p<0.01 ; I^{2}=90.89 \%$ ), and 36.63 (95\% CI: 25.38-52.87, $\left.p<0.01 ; I^{2}=100 \%\right)$, respectively. The optimal cut-off values of TE were $7.25 \mathrm{kPa}$ for diagnosing significant fibrosis and $12.4 \mathrm{kPa}$ for diagnosing cirrhosis, respectively. Conclusion. TE is of great value in the detection of patients with CHB-related cirrhosis but has a suboptimal accuracy in the detection of significant fibrosis.

\section{Introduction}

Chronic hepatitis B virus infection continues to be a major public health issue worldwide with the prevalence of $3.61 \%$ [1]. As well known, liver fibrosis, one of the main prognostic factors in chronic hepatitis B (CHB), was associated with the risk of developing cirrhosis and cirrhosis-related complications $[2,3]$. Therefore, liver fibrosis stage plays one of the most important roles in diagnostic and prognostic assessments in patients with $\mathrm{CHB}$.

Liver biopsy (LB), as invasive in nature with related risks, is the gold standard for fibrosis assessment. However, LB is associated with obvious patient discomfort and risk of complications ranging from pain to more serious events with hospitalization rate of $1.4-3.2 \%$ [4] and mortality varying from 0.0088 to $0.3 \%$ [5]. Besides, LB provides only a quite small part of the organ, and thus there is a risk that the small part might not be representative for the live fibrosis in the whole liver [6].

Noninvasive methods of assessing fibrosis and cirrhosis were urgently needed, and serologic tests and novel imaging techniques were recently developed $[7,8]$. Most of these studied focused on whether noninvasive methods can accurately detect minimal (F0-1), significant $(\geq F 2)$, or advanced ( $\geq$ F3-4) fibrosis based on the METAVIR score [9]. Transient elastography (TE), also known as FibroScan, was 
a device and a well-validated method with advantages of a short procedure time $(<5 \mathrm{~min})$, immediate results, and the ability to perform the test at the bedside or in an outpatient clinic [10]. Compared with blood tests, TE has a similar performance to predict significant fibrosis (SF) and higher accuracy to identify cirrhosis [11]. Measurement of liver fibrosis without biopsy is very tempting. In spite of the fact that recommendations suggested that noninvasive tests were still not ready to replace LB $[12,13]$, TE has become widely present in clinical practice. The accuracy of TE for detection of fibrosis has been assessed extensively in a variety of liver diseases [14-17]. However, it was reported that the presence of an IQR/M $>30 \%$ and liver stiffness median $\geq 7.1 \mathrm{kPa}$ lead to a lower accuracy determined by the area under receiveroperating curve (AUROC) and these cases were considered "poorly reliable" [18]. Another study also indicated that there was a significant discrepancy in up to $20 \%$ of cases cirrhosis between different TE devices [19].

In the study, we performed an independent meta-analysis of the diagnostic accuracy of TE for predicting significant liver fibrosis (F2-4 versus F0-1) and cirrhosis (F4 versus $\mathrm{F} 0-3)$ in $\mathrm{CHB}$ patients.

\section{Methods}

2.1. Literature Search Strategy. PubMed, Web of Science, and EMBASE database were searched to October 10, 2016, as well as Wanfang database and China National Knowledge Infrastructure. The search strategy was "FibroScan or transient elastography" in combination with "liver fibrosis assessment," "significant fibrosis or cirrhosis or advanced liver fibrosis," and "liver stiffness measurement." All eligible studies were retrieved and their reference lists were checked for additional relevant publications.

2.2. Inclusion Criteria. All diagnostic cross-sectional studies, cohort studies, and randomized studies that compared TE accuracy with biopsy in diagnosis fibrosis grade were eligible for inclusion. Studies that met all the following criteria were included: (i) studies which reported that all patients had undergone biopsy and TE; (ii) having enough data to create 2 $\times 2$ table of test performance (with numbers of true and false positives and negatives); and (iii) studies which reported the method of definition of the fibrosis grade.

2.3. Exclusion Criteria. The exclusion criteria were as follows: (i) the patients belonging to the pediatric population, hepatitis $\mathrm{C} /$ hepatitis $\mathrm{B}$ virus coinfected patients, mixed chronic liver disease patients (but not $\mathrm{CHB}$ and nonalcoholic fatty liver disease), and liver/kidney transplant patients; (ii) studies that were clearly extensions of previously published cohorts; and (iii) studies unable to obtain sufficient data for statistical analysis.

2.4. Methodological Assessment. Methodological quality was assessed by the Quality Assessment of Diagnostic Accuracy Studies-2 (QUADAS-2) tool. QUADAS-2 was designed to assess the internal and external validity. Any differences between two authors were resolved with discussion between the two review authors and the third author was final arbiter.
2.5. Data Extraction and Management. As for each study, the following information was extracted: year of publication, study design, sample size, presence of HIV coinfection, the QUADAS-2 methodological items, prevalence of each fibrosis stage on biopsy, along with total prevalence of SF and cirrhosis, interval between biopsy and TE, size of biopsy sample, type of scoring system used for histology (METAVIR versus other), and AUROC. Two authors performed the data extraction independently. Disagreement was resolved with discussion between the two review authors, with a third author as final arbiter.

2.6. Statistical Analysis and Data Synthesis. Initial analysis was performed with the Review Manager (RevMan) 5.0. Stata 12.0 was used for meta-analysis of diagnostic accuracy studies, to compute the pooled sensitivity and specificity and to plot the summary receiver-operating characteristics curve (SROC) with summary point and corresponding 95\% confidence interval (CI). Regression analysis was performed by Stata 12.0, with each time point providing another covariate to verify the influence of the chosen covariate on the accuracy estimates. We used hierarchical SROC model and the bivariate random efforts model to produce SROC and pooled estimates of sensitivity and specificity. We performed Fagan test to detect clinical significant by Stata 12.0. Heterogeneity was assessed with the inconsistency index $\left(I^{2}\right)$ and $I^{2}$ values over $50 \%$ indicated substantial heterogeneity. Heterogeneity from threshold effect was explored by meta-disc 1.4 .

\section{Results}

3.1. Search Results. 1238 articles were obtained and 188 were excluded for duplicates. 882 were excluded based on title and abstracts, and full-text copies of 106 studies were obtained and assessed for eligibility. Furthermore, 62 were excluded for inappropriate methodology, duplicate sample, pediatric population, or inability to obtain data for at least $2 \times 2$ table. Finally, a total of 44 articles comprising 45 studies were enrolled in the meta-analysis (Figure 1).

3.2. Characteristics of Included Studies. The overall prevalence of SF (F2-4) and cirrhosis (F4) ranged from $14.8 \%$ to $92.3 \%$ and from $1.1 \%$ to $69.2 \%$, respectively. Reported AUROCs for SF diagnosis ranged from 0.614 to 0.98 (Table 1).

As shown in Table 1, only Miailhes et al. $(N=59)$ reported HIV coinfected patients [20]. In sixteen studies $(N=2664)$, LB was assessed with a histological score other than METAVIR [21-36]. In eight studies $(N=1109)$, mean length of biopsy sample was $\geq 20 \mathrm{~mm}[22,34,37-42]$. Besides, in nineteen studies $(N=1358)$, data on time interval between biopsy and TE were not obtained $[11,21,23,25,27,28,32-$ $34,39,40,42-47]$. Three studies did not report cirrhosis (F4) $[24,35,48]$. Only four studies were retrospective [31, 48-50].

As presented in Figure 2, the results of methodological quality assessment based on the QUADAS-2 scale were depicted for all of the 44 eligible studies. The majority of the methodological concern lies within the index test, because TE in ten studies interpreted with knowledge of the results of the biopsy $[24,29,33,39,46,48,51-54]$ and TE in one study 


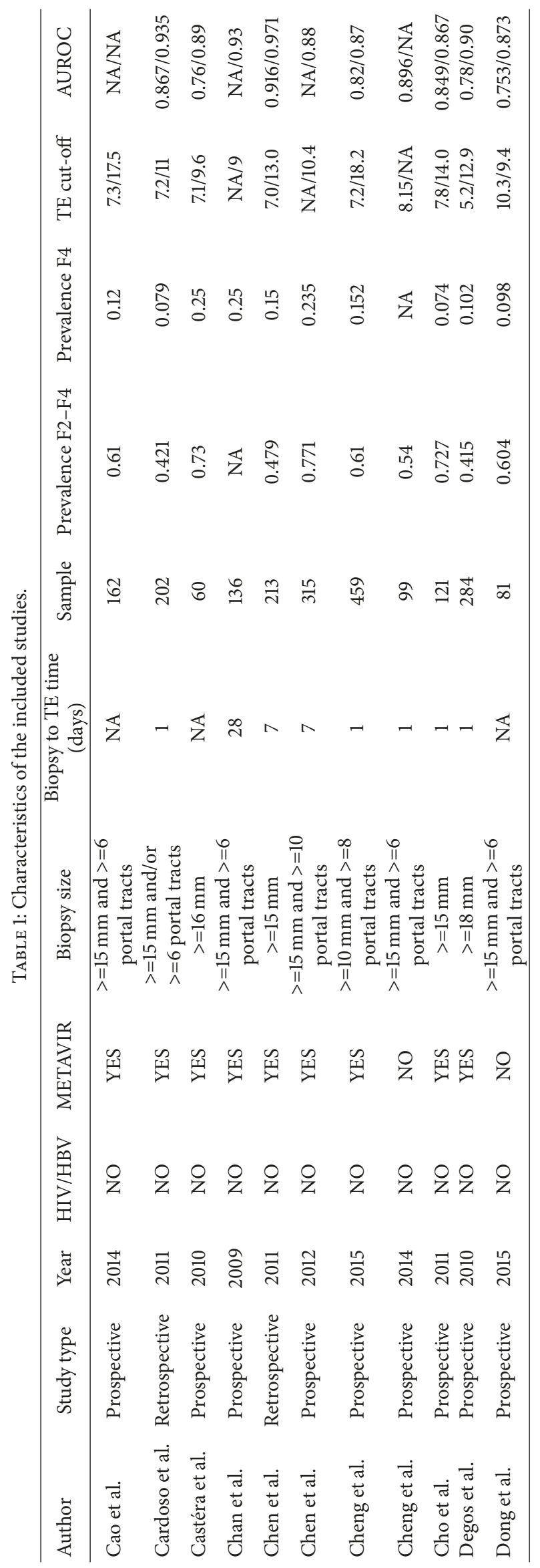




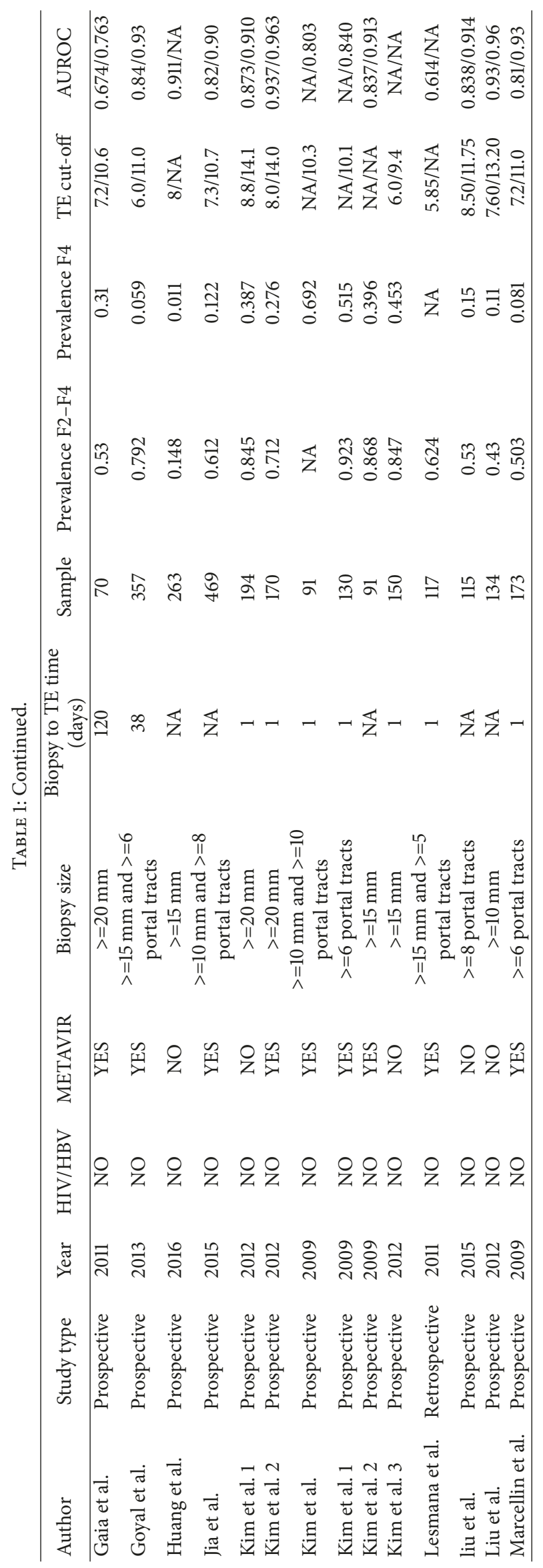




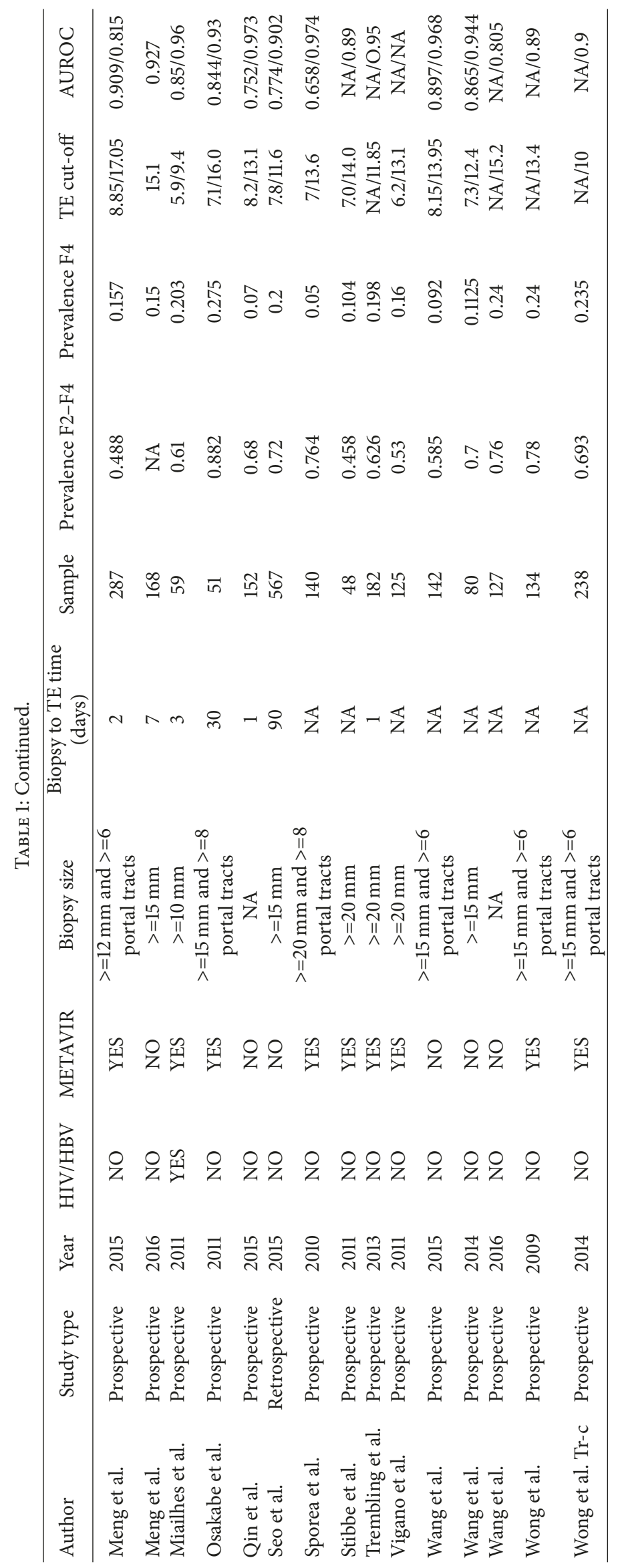




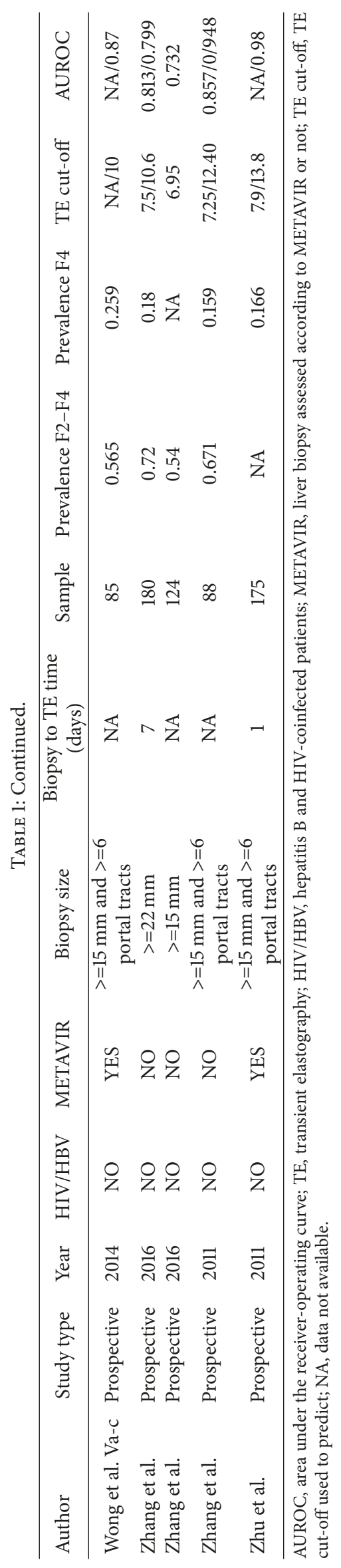




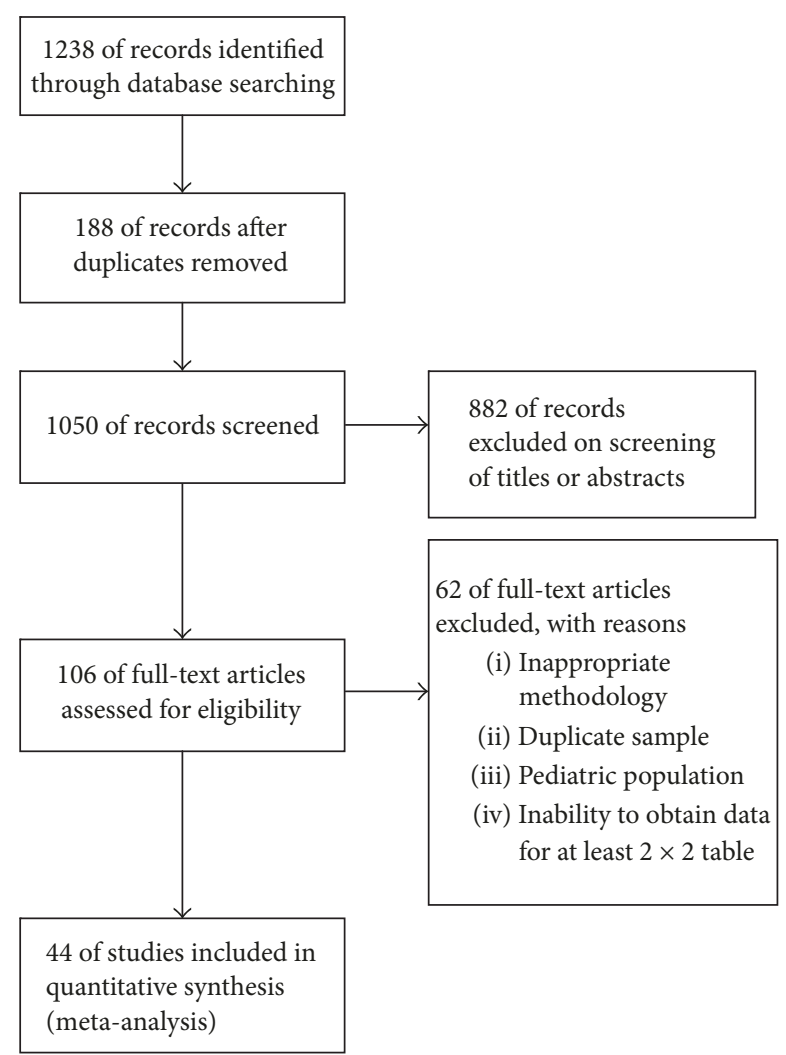

FIGURE 1: Flow diagram of study selection process.

was conducted with assistance by a time-motion ultrasound image [40]. Another possible issue was addressed in patient selection that participants might be enrolled consecutively with confirmed diagnosis in three studies [31, 50, 55]. Both of these concerns might be located in heterogeneity and sensitivity analyses.

3.3. Diagnosis of SF. We included 35 studies $(N=6,202)$ in the analysis for SF (F2-F4) [15-23, 25-27, 29-35, 37-40, $43,56-59]$. Summary representation of the overall analysis was presented in Figure 3(a) and Supplementary Figure 1. Sensitivity and specificity ranged from 51 to $97 \%$ and 38 to $100 \%$, respectively (Supplementary Figure 1).

The area under SROC for SF was 0.86 (95\% CI: 0.83-0.89) (Figure 3(a)). The meta-analysis summary estimate indicated pooled sensitivity of 0.78 (95\% CI: $0.73-0.81, p<0.01 ; I^{2}=$ $85.59 \%$ ), specificity of 0.81 (95\% CI: $0.77-0.84, p<0.01$; $I^{2}=88.20 \%$ ) (Supplementary Figure 1(A)), positive likelihood ratio (LR+) of 4.01 (95\% CI: 3.31-4.84, $p<0.01 ; I^{2}=$ $86.27 \%$ ), negative likelihood ratio (LR-) of 0.28 (95\% CI: $0.23-0.33, p<0.01 ; I^{2}=81.95 \%$ ) (Supplementary Figure 1(B)), diagnostic score (DS) of 2.67 (95\% CI: 2.38-2.96, $p<$ $0.01 ; I^{2}=71.57 \%$ ), and diagnostic odds ratio (DOR) of 14.44 (95\% CI: $10.80-19.30, p<0.01 ; I^{2}=100 \%$ ) (Supplementary Figure $1(\mathrm{C})$ ). However, it must be carefully considered as they were not pooled from studies with identical TE threshold. Overall, there was heterogeneity as graphically illustrated on the forest plot in Supplementary Figure 1. The cut-off value for SF (F2-4) ranged from 5.2 to $10.3 \mathrm{kPa}$ with a mean value of $8.6 \mathrm{kPa}$ and a median of $7.25 \mathrm{kPa}$.

As shown in Figure 3(b) and Table 2, in the analysis of LB-related factors with an impact on accuracy, there was no significant difference (joint $p=0.47$ for classification criteria; joint $p=0.29$ for interval time; joint $p=0.77$ for average sample size). 26 studies conducted in Asian presented a better both pooled sensitivity $(0.78,95 \% \mathrm{CI}: 0.73-0.82)$ and specificity (0.83, 95\% CI: $0.79-0.87)$ than in Caucasian (joint $p=0.03)$.

As presented in Figure 3(c), it was indicated that posttest probability of LR+ increased to $86 \%$ and LR- decreased to $29 \%$ after TE was performed based on Fagan test.

3.4. Diagnosis of Cirrhosis. 41 studies were included in the cirrhotic analysis with a total of 7,205 patients, as four studies did not have any cases of liver cirrhosis (METAVIR F4) $[21,24,35,48]$. The overall prevalence of METAVIR F4 and the AUROCs in the included studies ranged from 5\% to $69.2 \%$ and from 0.80 to 0.98 (Table 1 ), respectively.

Summary representation of the overall analysis was shown in Figure 4(a). The area under the SROC for liver cirrhosis was 0.92 (95\% CI: 0.90-0.94). Sensitivity ranged from $49 \%$ to $100 \%$, much more widely than specificity which ranged from $62 \%$ to $99 \%$ (Supplementary Figure 2). The meta-analysis summary estimate covered the pooled sensitivity of 0.84 (95\% CI: $0.80-0.88, p<0.01 ; I^{2}=76.67 \%$ ), specificity of 0.87 (95\% CI: $0.84-0.90, p<0.01 ; I^{2}=90.89 \%$ ) (Supplementary Figure 2(A)), LR+ of 6.66 (95\% CI: 5.34-8.31, $\left.p<0.01 ; I^{2}=84.77 \%\right)$, LR- of 0.18 (95\% CI: $0.14-0.23, p<$ $0.01 ; I^{2}=80.80 \%$ ) (Supplementary Figure 2(B)), DS of 3.60 (95\% CI: 3.23-3.97, $p<0.01 ; I^{2}=66.54 \%$ ), and DOR of 36.63 (95\% CI: 25.38-52.87, $p<0.01 ; I^{2}=100 \%$ ), respectively (Supplementary Figure 2(C)). Again, these measures must be carefully considered without identical TE thresholds. The cut-off value for cirrhosis ranged from $9 \mathrm{kPa}$ to $18.2 \mathrm{kPa}$ with both a mean value and a median of $12.4 \mathrm{kPa}$.

As shown in Figure 4(b) and Table 3, although summary sensitivity was lower and summary specificity was higher in studies with METAVIR score (sensitivity: 0.82, 95\% CI: 0.77-0.87; specificity: 0.88, 95\% CI: 0.85-0.91), TE performed on the next day of LB (sensitivity: $0.79,95 \%$ CI: $0.71-0.86$; specificity: $0.88,95 \%$ CI: $0.84-0.93$ ), and average sample length $\geqslant 20 \mathrm{~mm}$ (sensitivity: 0.79, 95\% CI: 0.69-0.89; specificity: $0.88,95 \%$ CI: $0.83-0.94)$, respectively, no statistical significance was detected (joint $p=0.17$ for classification criteria; joint $p=0.21$ for interval time; joint $p=0.47$ for average sample size). Besides, pooled sensitivity and specificity were without significant difference (joint $p=$ 0.12) between Caucasian (sensitivity: 0.78, 95\% CI: 0.67-0.88; specificity: 0.91, 95\% CI: 0.86-0.95) and Asian (sensitivity: 0.86, 95\% CI: 0.81-0.90; specificity: 0.86, 95\% CI: 0.83-0.89).

In addition, based on Fagan test, it was illustrated that posttest probability of LR+ and LR- rose and declined to $59 \%$ and $4 \%$, respectively (Figure $4(\mathrm{c})$ ).

3.5. Publication Bias. The results of publication bias analysis were performed with Stata in Supplementary Figure 3. No 


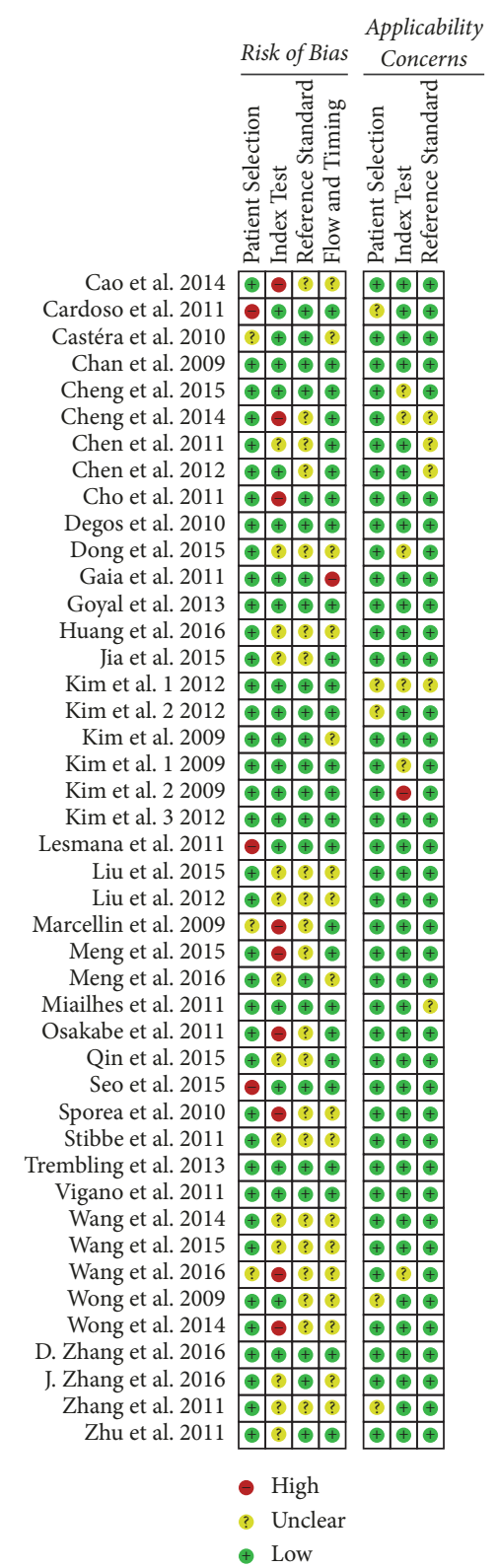

(a)

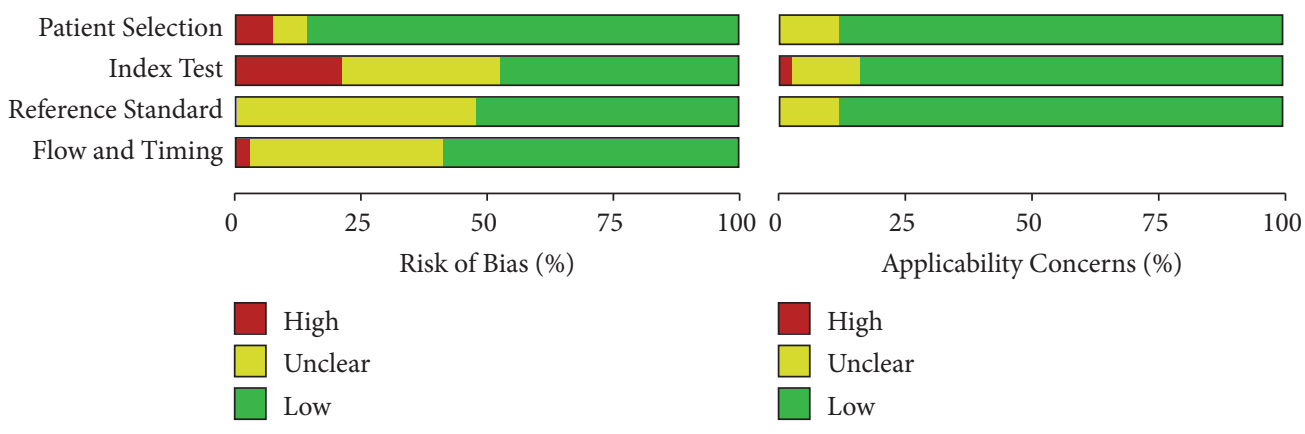

(b)

FIGURE 2: Summary of methodological quality of 44 studies according to Quality Assessment of Diagnostic Studies-2 (QUDAS-2) tool. (a) Overall and (b) study-level of bias. 


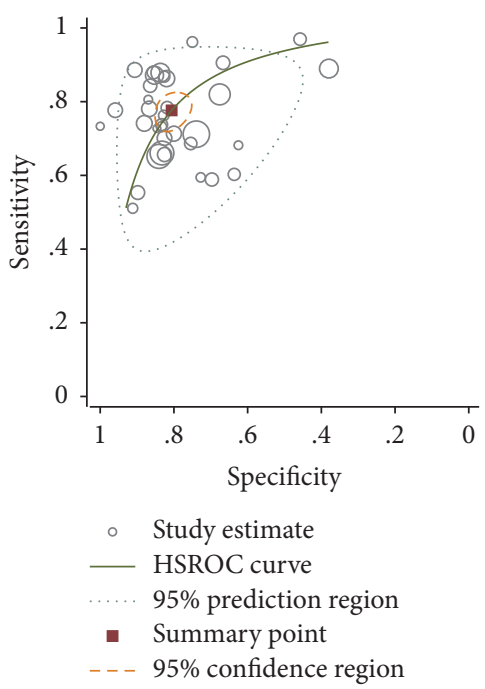

(a)

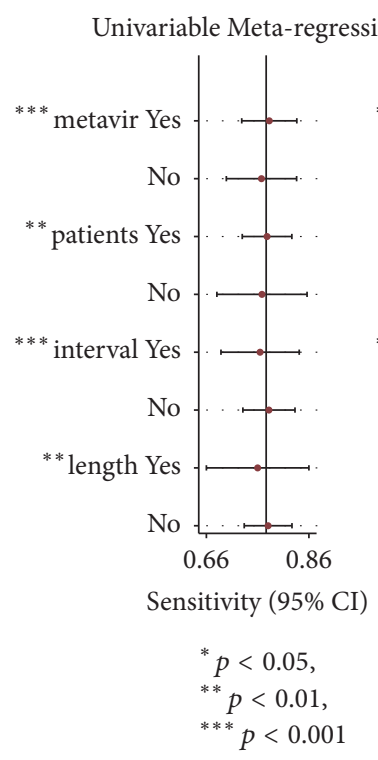

(b)

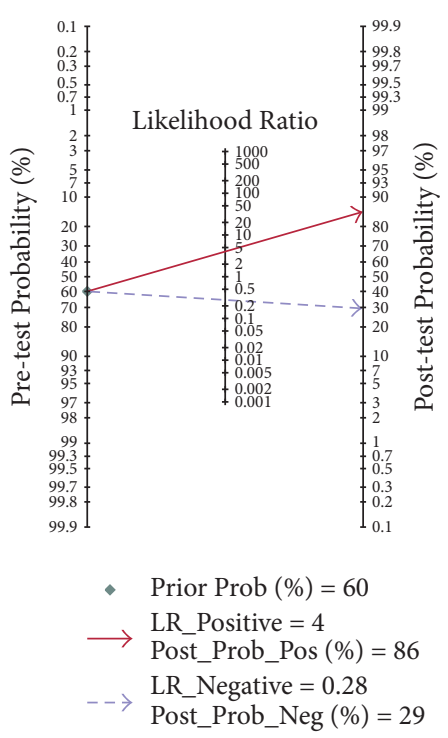

(c)

FIGURE 3: Meta-analysis of 32 studies that assessed the diagnosis accuracy of significant fibrosis based on transient elastography. (a) A summary receiver-operating characteristic (SROC) plot of transient elastography for detection of significant liver fibrosis (METAVIR F2-F4). (b) Regression analysis of studies whether reported with METAVIR score on the next day of biopsy or with sample size $\geq 20 \mathrm{~cm}$ for significant liver fibrosis. (c) Detection of clinical significance for significant liver fibrosis (METAVIR F2-F4) based on Fagan test. Heterogeneity was generated if $p<0.01$ in sensitivity or specificity separately. However, joint $p$ value was generated synthesisly for analysis of both sensitivity and specificity.

TABLE 2: Results of meta-regression for significant fibrosis.

\begin{tabular}{|c|c|c|c|c|c|c|}
\hline Covariate & Number & Pooled sensitivity & $p$ value & Pooled specificity & $p$ value & Joint $p$ value \\
\hline \multicolumn{7}{|l|}{ Classification criteria } \\
\hline METAVIR score & 21 & $0.78(0.75-0.83)$ & \multirow{2}{*}{$<0.01$} & $0.79(0.73-0.84)$ & \multirow{2}{*}{$<0.01$} & \multirow{2}{*}{0.47} \\
\hline Non-METAVIR score & 14 & $0.77(0.70-0.83)$ & & $0.83(0.78-0.89)$ & & \\
\hline \multicolumn{7}{|l|}{ Interval time } \\
\hline On the next day of liver biopsy & 11 & $0.76(0.69-0.84)$ & \multirow{2}{*}{$<0.01$} & $0.85(0.79-0.90)$ & \multirow{2}{*}{$<0.01$} & \multirow{2}{*}{0.29} \\
\hline More than one day after liver biopsy & 24 & $0.78(0.73-0.83)$ & & $0.78(0.74-0.83)$ & & \\
\hline \multicolumn{7}{|l|}{ Average sample size } \\
\hline$\geqslant 20 \mathrm{~mm}$ & 7 & $0.76(0.66-0.86)$ & \multirow{2}{*}{$<0.01$} & $0.79(0.69-0.88)$ & \multirow{2}{*}{$<0.01$} & \multirow{2}{*}{0.77} \\
\hline Not $\geqslant 20 \mathrm{~mm}$ & 28 & $0.78(0.74-0.82)$ & & $0.81(0.77-0.85)$ & & \\
\hline \multicolumn{7}{|l|}{ Region } \\
\hline Asian & 26 & $0.78(0.73-0.82)$ & \multirow{2}{*}{$<0.01$} & $0.83(0.79-0.87)$ & \multirow{2}{*}{0.04} & \multirow{2}{*}{0.03} \\
\hline Caucasian & 9 & $0.77(0.68-0.85)$ & & $0.72(0.63-0.80)$ & & \\
\hline
\end{tabular}

significant publication bias was detected according to Deeks figures for SF ( $p=0.26)$. However, there was bias among 41 studies enrolled in analysis of TE for cirrhosis $(p=0.02)$, which might result from the positive results of all 41 studies.

\section{Discussion}

TE can provide a reliable detection of liver fibrosis in patients with $\mathrm{CHB}$ and thus has been recommended by the American Association for the Study of Liver Diseases (AASLD) and European Association for the Study of the Liver (EASL) $[60,61]$. This meta-analysis was conducted in a total of 7,808 $\mathrm{CHB}$ patients to summarize the diagnostic accuracy of $\mathrm{TE}$ for CHB-related SF, with optimal statistical method SROC. In addition, regression analysis was carried out to further explore sources of heterogeneity.

In our study, TE performed well in both SF (F2-4) and cirrhosis (F4) with pooled sensitivity of $78 \%$ and $84 \%$, summary specificity of $81 \%$ and $87 \%$, DOR of 14.44 and 36.63 , LR+ of 4.01 and 6.66, LR- of 0.28 and 0.18 , respectively. Study by $\mathrm{Li}$ et al. [62] with hierarchical SROC model was also performed in $\mathrm{CHB}$ patients, with summary sensitivity and specificity for SF (F2-4) and cirrhosis (F4) of $80 \%$ and $86 \%, 82 \%$, and $88 \%$, however, without DOR, LR+ and LR-. Interestingly, the pooled specificity for diagnosis SF (F2-4) and cirrhosis (F4) in both studies were higher than 


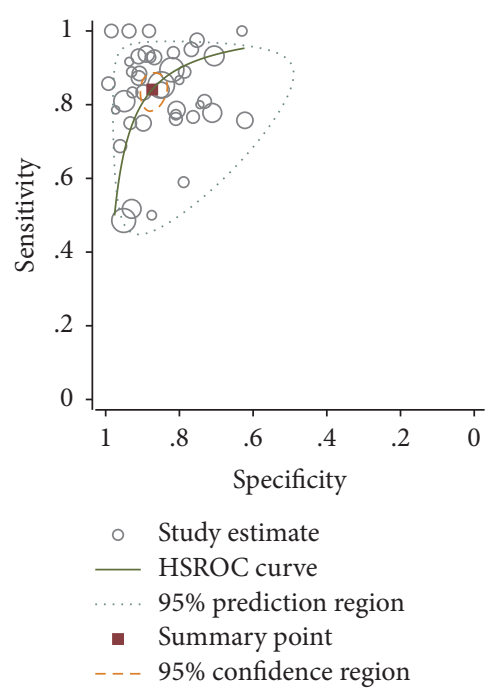

(a)

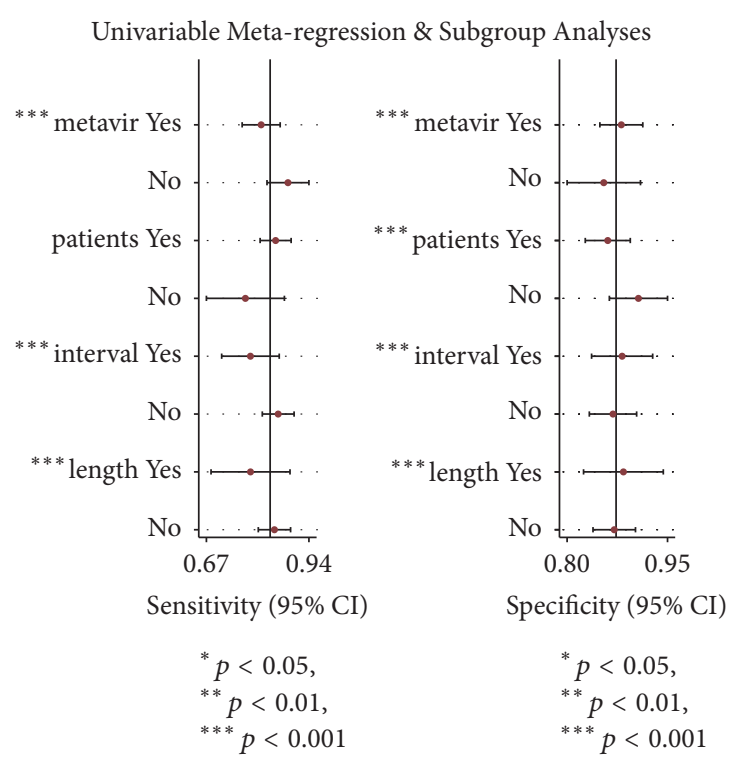

(b)

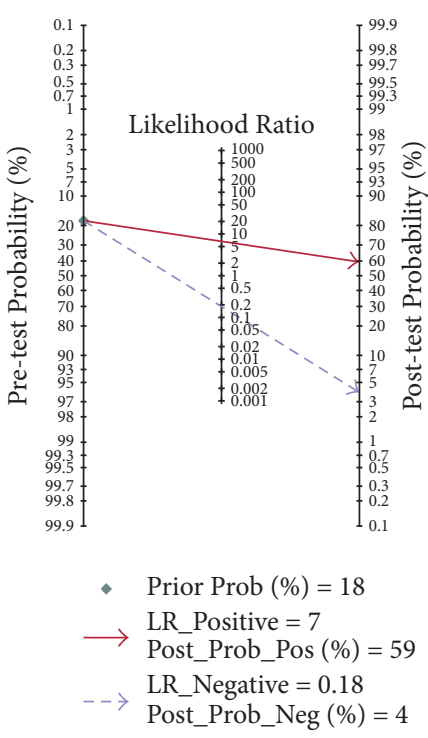

(c)

FIGURE 4: Meta-analysis of 37 studies that assessed the diagnosis accuracy of cirrhosis based on transient elastography. (a) A summary receiver-operating characteristic (SROC) plot of transient elastography for detection of cirrhosis (METAVIR F4). (b) Regression analysis of studies whether reported with METAVIR score on the next day of biopsy or with sample size $\geq 20 \mathrm{~cm}$ for cirrhosis. (c) Detection of clinical significance for cirrhosis (METAVIR F4) based on Fagan test.

TABLE 3: Results of meta-regression for cirrhosis.

\begin{tabular}{|c|c|c|c|c|c|c|}
\hline Covariate & Number & Pooled sensitivity & $p$ value & Pooled specificity & $p$ value & Joint $p$ value \\
\hline \multicolumn{7}{|l|}{ Classification criteria } \\
\hline METAVIR score & 28 & $0.82(0.77-0.87)$ & \multirow{2}{*}{$<0.01$} & $0.88(0.85-0.91)$ & \multirow{2}{*}{$<0.01$} & \multirow{2}{*}{0.17} \\
\hline Non-METAVIR score & 13 & $0.89(0.83-0.94)$ & & $0.86(0.80-0.91)$ & & \\
\hline \multicolumn{7}{|l|}{ Interval time } \\
\hline On the next day of liver biopsy & 13 & $0.79(0.71-0.86)$ & \multirow{2}{*}{$<0.01$} & $0.88(0.84-0.93)$ & \multirow{2}{*}{$<0.01$} & \multirow{2}{*}{0.21} \\
\hline More than one day after liver biopsy & 28 & $0.86(0.82-0.90)$ & & $0.87(0.83-0.90)$ & & \\
\hline \multicolumn{7}{|l|}{ Average sample size } \\
\hline$\geqslant 20 \mathrm{~mm}$ & 8 & $0.79(0.69-0.89)$ & \multirow{2}{*}{$<0.01$} & $0.88(0.83-0.94)$ & \multirow{2}{*}{$<0.01$} & \multirow{2}{*}{0.47} \\
\hline Not $\geqslant 20 \mathrm{~mm}$ & 33 & $0.85(0.81-0.89)$ & & $0.87(0.84-0.90)$ & & \\
\hline \multicolumn{7}{|l|}{ Region } \\
\hline Asian & 31 & $0.86(0.81-0.90)$ & \multirow{2}{*}{$<0.01$} & $0.86(0.83-0.89)$ & \multirow{2}{*}{$<0.01$} & \multirow{2}{*}{0.12} \\
\hline Caucasian & 10 & $0.78(0.67-0.88)$ & & $0.91(0.86-0.95)$ & & \\
\hline
\end{tabular}

summary sensitivity, which suggested that the currently cutoff values of TE performed better in excluding diseases rather than confirming diseases. Furthermore, the areas under the SROC were 0.86 for SF (F2-4) and 0.92 for cirrhosis (F4), respectively, which indicated that TE was performed well in staging fibrosis in $\mathrm{CHB}$ patients. In addition, TE performed better for cirrhosis than SF with a higher value of AUC, sensitivity, specificity, DOR, LR+, and a lower value of LR-. Although the diagnostic accuracy was higher for cirrhosis, TE could also increase the diagnostic accuracy for SF based on Fagan test with increased LR+ and decreased LR-.

The higher TE values were used to confirm diagnosis, while the lower one was used to exclude the false positive diagnosis. However, if the TE value located between the values for rule in and rule out, biopsy was then recommended. Based on the descriptive statistics of enrolled studies, the cut-off values for diagnosing SF (F2-4) and cirrhosis (F4) ranged from 5.2 to $10.3 \mathrm{kPa}$ and 9 to $18.2 \mathrm{kPa}$, respectively. The optimal cut-off values of TE in CHB patients in our study were $7.25 \mathrm{kPa}$ for SF (F2-4) and $12.4 \mathrm{kPa}$ for cirrhosis (F4). In the previous meta-analysis by Li et al., the weighted mean cut-off values of TE were comparable with $7.2 \mathrm{kPa}$ for $\mathrm{SF}$ (F2-4) and $12.2 \mathrm{kPa}$ for cirrhosis (F4) [62]. However, since there was no optimal statistical method to pool different cutoff values in individual studies, the optimal cut-off values in our meta-analysis were simply summarized as median, which could eliminate the impact resulting from the maximum and 
minimum values that was better than the mean value in previous study [62].

Elevated ALT levels might affect the predictive accuracy of TE $[16,24,45,50,55,56]$; however, the study by Cardoso et al. reported that the use of TE cut-off values adjusted to ALT level did not improve the performance of liver stiffness in CHB patients [49]. Although elevated ALT might be the most important confounder on liver stiffness measurement, the synthesis analysis of ALT elevation could not be conducted due to insufficient data. Therefore, it would be beneficial if more clinical studies focused on the correlation between ALT elevation and TE in CHB patients.

One of the main limitations in this meta-analysis was the significant heterogeneity of the included studies. Spearman correlation coefficient for SF and cirrhosis were 0.055 ( $p=$ $0.755)$ and $0.057(p=0.723)$, and no threshold effect was presented. Therefore, regression analysis was carried out. Besides, TE value could be applied as diagnosis criteria for both SF and cirrhosis in Asian. However, for Caucasian, it was noted that TE was valid to diagnosis of cirrhosis, while it was less precise for SF. Unfortunately, the regression analysis was not conducted owing to the small size of HIV- and non-HIVcoinfected patients. It should be noted that the overlapped cut-off values from included studies might also result in the heterogeneity.

In conclusion, TE is of great value for detection CHBrelated cirrhosis, however, with a suboptimal performance in detection of SF. Further studies should focus on the TE cut-off value and the effect of ALT elevation in patients with CHB.

\section{Abbreviations}

$\begin{array}{ll}\text { CHB: } & \text { Chronic hepatitis B } \\ \text { CI: } & \text { Confidence interval } \\ \text { DOR: } & \text { Diagnostic odds ratio } \\ \text { LB: } & \text { Liver biopsy } \\ \text { LR: } & \text { Likelihood ratio } \\ \text { QUADAS-2: } & \text { Quality Assessment of Diagnostic } \\ & \text { Accuracy Studies-2 } \\ \text { SF: } & \text { Significant fibrosis } \\ \text { SROC: } & \text { Summary receiver-operating curves } \\ \text { TE: } & \text { Transient elastography } \\ \text { AUROC: } & \text { Area under receiver-operating curve. }\end{array}$

\section{Conflicts of Interest}

The authors have no relevant affiliations or financial involvement with any organization or entity with a financial interest in or financial conflict with the subject matter or materials discussed in the manuscript. This includes employment, consultancies, honoraria, stock ownership or options, expert testimony, grants or patients received or pending, or royalties.

\section{Authors' Contributions}

Xiaolong Qi, Weidong Wang, Jing Wang, and CHESS Study Group contributed to study concepts and design; Min An and Tongwei Wu performed literature search; Min An and Jing Wang conducted data extraction; Min An, Tongwei Wu, Deke
Jiang, Mengyun Peng, and Chunqing Zhang performed data analysis: Tongwei Wu, Weidong Wang, Chunqing Zhang, and CHESS Study Group were responsible for manuscript preparation and revision. All authors and CHESS Study Group have participated sufficiently in the study and approved the final version. Xiaolong Qi, Min An, and Tongwei Wu contributed equally to this work.

\section{Acknowledgments}

Collaborators of CHESS Study Group are as follows: Zhiwei Li, Department of General Surgery, 302 Hospital of PLA, Beijing, China; Fuquan Liu, Department of Interventional Therapy, Beijing Shijitan Hospital, Capital Medical University, Beijing, China; Guofeng Chen, Liver Cirrhosis Diagnosis and Treatment Center, 302 Hospital of PLA, Beijing, China; and Qingge Zhang, Department of Hepatology, Xingtai People's Hospital, Xingtai, China. This work was supported by the grants from the National Natural Science Foundation of China (81600510), Guangzhou Industry-Academia-Research Collaborative Innovation Major Project (201704020015), and President Foundation of Nanfang Hospital, Southern Medical University (2017Z012).

\section{Supplementary Materials}

Supplementary Figure 1: meta-analysis of 32 studies that assessed the diagnosis accuracy of significant fibrosis (METAVIR F2-F4) based on transient elastography. A Forest plot of (A) sensitivity and specificity, (B) positive and negative likelihood ratio, and (C) diagnostic score (DS) and diagnostic odds ratio (DOR) for significant liver fibrosis (METAVIR F2-F4). Supplementary Figure 2: meta-analysis of 37 studies that assessed the diagnosis accuracy of cirrhosis (METAVIR F4) based on transient elastography. A Forest plot of (A) sensitivity and specificity, (B) positive and negative likelihood ratio, and (C) DS and DOR for cirrhosis (METAVIR F4). Supplementary Figure 3: Deeks' Funnel Plot Asymmetry Test for (A) significant fibrosis (METAVIR F2-F4) and (B) cirrhosis (METAVIR F4). (Supplementary Materials)

\section{References}

[1] A. Schweitzer, J. Horn, R. T. Mikolajczyk, G. Krause, and J. J. Ott, "Estimations of worldwide prevalence of chronic hepatitis $B$ virus infection: a systematic review of data published between 1965 and 2013," The Lancet, vol. 386, no. 10003, pp. 1546-1555, 2015.

[2] X. Lu, X. Li, Z. Yuan et al., "Assessment of liver fibrosis with the gamma-glutamyl transpeptidase to platelet ratio: a multicentre validation in patients with HBV infection," Gut, 2017.

[3] X. Qi, X. Zhang, Z. Li et al., "HVPG signature: A prognostic and predictive tool in hepatocellular carcinoma," Oncotarget , vol. 7, no. 38, pp. 62789-62796, 2016.

[4] C. H. Janes and K. D. Lindor, "Outcome of patients hospitalized for complications after outpatient liver biopsy," Annals of Internal Medicine, vol. 118, no. 2, pp. 96-98, 1993.

[5] D. H. Van Thiel, J. S. Gavaler, H. Wright, and A. Tzakis, "Liver biopsy: Its safety and complications as seen at a liver transplant center," Transplantation, vol. 55, no. 5, pp. 1087-1090, 1993. 
[6] A. Regev, M. Berho, L. J. Jeffers et al., "Sampling error and intraobserver variation in liver biopsy in patients with chronic $\mathrm{HCV}$ infection," American Journal of Gastroenterology, vol. 97, no. 10, pp. 2614-2618, 2002.

[7] G. Shiha, A. Ibrahim, A. Helmy et al., "Asian-Pacific Association for the Study of the Liver (APASL) consensus guidelines on invasive and non-invasive assessment of hepatic fibrosis: a 2016 update," Hepatology International, vol. 11, no. 1, 2017.

[8] X. Qi, Z. Li, J. Huang et al., "Virtual portal pressure gradient from anatomic CT angiography," Gut, vol. 64, no. 6, pp. 10041005, 2015.

[9] M. Pinzani, F. Vizzutti, U. Arena, and F. Marra, “Technology Insight: noninvasive assessment of liver fibrosis by biochemical scores and elastography," Nature Clinical Practice Gastroenterology \& Hepatology, vol. 5, no. 2, pp. 95-106, 2008.

[10] European Association for Study of Liver and Asociacion Latinoamericana para el Estudio del Higado, "EASL-ALEH Clinical Practice Guidelines: non-invasive tests for evaluation of liver disease severity and prognosis," Journal of Hepatology, vol. 63, no. 1, pp. 237-264, 2015.

[11] L. Castéra, J. Vergniol, J. Foucher et al., "Prospective comparison of transient elastography, fibrotest, APRI, and liver biopsy for the assessment of fibrosis in chronic hepatitis C," Gastroenterology, vol. 128, no. 2, pp. 343-350, 2005.

[12] G. Sebastiani and A. Alberti, "Non invasive fibrosis biomarkers reduce but not substitute the need for liver biopsy," World Journal of Gastroenterology, vol. 12, no. 23, pp. 3682-3694, 2006.

[13] D. C. Rockey and D. M. Bissell, "Noninvasive measures of liver fibrosis," Hepatology, vol. 43, no. 2, pp. S113-S120, 2006.

[14] S. Singh, L. L. Fujii, M. H. Murad et al., "Liver stiffness is associated with risk of decompensation, liver cancer, and death in patients with chronic liver diseases: a systematic review and meta-analysis," Clinical Gastroenterology and Hepatology, vol. 11, no. 12, pp. 1573-1584, 2013.

[15] A. Berzigotti, M. Reig, J. G. Abraldes, J. Bruix, J. Bosch, and J.-C. García-Pagán, "Value of transient elastography measured with fibroscan in predicting the outcome of hepatic resection for hepatocellular carcinoma," Annals of Surgery, vol. 261, no. 4, p. e105, 2015.

[16] C. Corpechot, A. El Naggar, A. Poujol-Robert et al., "Assessment of biliary fibrosis by transient elastography in patients with PBC and PSC," Hepatology, vol. 43, no. 5, pp. 1118-1124, 2006.

[17] C. S. Pavlov, G. Casazza, D. Nikolova, E. Tsochatzis, and C. Gluud, "Systematic review with meta-analysis: Diagnostic accuracy of transient elastography for staging of fibrosis in people with alcoholic liver disease," Alimentary Pharmacology \& Therapeutics, vol. 43, no. 5, pp. 575-585, 2016.

[18] J. Boursier, J.-P. Zarski, V. de Ledinghen et al., "Determination of reliability criteria for liver stiffness evaluation by transient elastography," Hepatology, vol. 57, no. 3, pp. 1182-1191, 2013.

[19] J. Parra-Ruiz, C. Sanjuán, L. Muñoz-Medina, D. Vinuesa, M. A. Martínez-Pérez, and J. Hernández-Quero, "Letter: Accuracy of liver stiffness measurement - A comparison of two different FibroScan devices," Alimentary Pharmacology \& Therapeutics, vol. 39, no. 12, pp. 1434-1435, 2014.

[20] P. Miailhes, P. Pradat, M. Chevallier et al., "Proficiency of transient elastography compared to liver biopsy for the assessment of fibrosis in HIV/HBV-coinfected patients," Journal of Viral Hepatitis, vol. 18, no. 1, pp. 61-69, 2011.

[21] R. Huang, N. Jiang, R. Yang et al., "Fibroscan improves the diagnosis sensitivity of liver fibrosis in patients with chronic hepatitis B," Experimental and Therapeutic Medicine, vol. 11, no. 5, pp. 1673-1677, 2016.

[22] B. K. Kim, S. U. Kim, H. S. Kim et al., "Prospective validation of Fibrotest in comparison with liver stiffness for predicting liver fibrosis in Asian subjects with chronic hepatitis B," PLoS ONE, vol. 7, no. 4, Article ID e35825, 2012.

[23] C. Wang, X. Cheng, C. Meng, and W. Lu, "Diagnostic value of Fibrotest for liver fibrosis in patients with chronic hepatitis," Chinese Journal of Hepatology, vol. 23, pp. 738-741, 2015.

[24] X. Cheng, W. Lu, W. Hou, C. Wang, Y. Liu, and J. Wang, "Diagnostic value of FibroTest combined FibroScan for liver fibrosis in patients with chronic hepatitis B," Journal of Clinical Hepatology, vol. 30, pp. 424-427, 2014.

[25] D.-R. Dong, M.-N. Hao, C. Li et al., "Acoustic radiation force impulse elastography, FibroScan ${ }^{\circledR}$, Forns' index and their combination in the assessment of liver fibrosis in patients with chronic hepatitis B, and the impact of inflammatory activity and steatosis on these diagnostic methods," Molecular Medicine Reports, vol. 11, no. 6, pp. 4174-4182, 2015.

[26] S. U. Kim, J. K. Kim, Y. N. Park, and K.-H. Han, "Discordance between liver biopsy and fibroscan ${ }^{\circledR}$ in assessing liver fibrosis in chronic hepatitis b: Risk factors and influence of necroinflammation," PLoS ONE, vol. 7, no. 2, Article ID e32233, 2012.

[27] D. Liu, Q. Yang, M. Zhang, L. Li, M. Li, and B. Zhao, "Value of Fibroscan in diagnosis of chronic hepatitis B liver fibrosis," China Practical Medicine, p. 10, 2015.

[28] Z. Liu, J. Feng, Q. Xiao, L. Ye, X. Wu, and R. Du, "Application of FibroScan for Diagnosis of Liver Fibrosis in Patients with Chronic Hepatitis B," Chinese General Prac, vol. 15, pp. 40684070, 2012.

[29] Y. Meng, H. Zhang, Z. Yu, H. Liang, and Z. Li, "Value of fibroscan in diagnosis of hepatic fibrosis in patients with chronic hepatitis B infection who had alanine ALT levels lower than 2 times the upper normal limit value," Chinese J Prac Med, pp. 4344, 2016.

[30] H. Qin and H. Yin, "Application of FibroScan combined with APRI for liver fibrosis in patients with chronic hepatitis B," Anhui Medical Journal, vol. 36, pp. 552-556, 2015.

[31] Y. S. Seo, M. Y. Kim, S. U. Kim et al., "Accuracy of transient elastography in assessing liver fibrosis in chronic viral hepatitis: A multicentre, retrospective study," Liver International, vol. 35, no. 10, pp. 2246-2255, 2015.

[32] C. Wang, J. Wang, B. Jia, C. Li, and C. Liu, "Diagnostic value of FibroScan for liver fibrosis in patients with chronic hepatitis B. Infect Dis Info," Infectious Disease Information, pp. 27-226, 2014.

[33] H. Wang, X. Xin, L. Zhang, Q. Ye, and Z. Ye, "Application value analysis of transient elastic wave monitoring in the development of chronic hepatitis," China Medical Equipment, pp. 13-61, 2016.

[34] D. Zhang, M. Chen, R. Wang et al., "Comparison of Acoustic Radiation Force Impulse Imaging and Transient Elastography for Non-invasive Assessment of Liver Fibrosis in Patients with Chronic Hepatitis B," Ultrasound in Medicine \& Biology, vol. 41, no. 1, pp. 7-14, 2015.

[35] J. Zhang, G. Li, S. Ma, and Y. Fang, "Comparative study of shear wave elastography and transient elastography on diagnosing significant liver fibrosis in patients with chronic hepatitis B," Modern Practical Medicine, pp. 28-288, 2016.

[36] X. Zhang, W. Lu, and C. Wang, "Diagnostic Value of Fibroscan for Liver Fibrosis in Patients with Chronic Hepatitis B. Tianjin Med," Tianjin Medical Journal, pp. 39-236, 2011. 
[37] S. Gaia, S. Carenzi, A. L. Barilli et al., "Reliability of transient elastography for the detection of fibrosis in Non-Alcoholic Fatty Liver Disease and chronic viral hepatitis," Journal of Hepatology, vol. 54, no. 1, pp. 64-71, 2011.

[38] B. K. Kim, H. S. Kim, J. Y. Park et al., "Prospective validation of ELF test in comparison with fibroscan and fibrotest to predict liver fibrosis in Asian subjects with chronic hepatitis B," PLoS ONE, vol. 7, no. 7, Article ID e41964, 2012.

[39] I. Sporea, R. Şirli, A. Deleanu et al., "Liver stiffness measurements in patients with HBV vs HCV chronic hepatitis: A comparative study," World Journal of Gastroenterology, vol. 16, no. 38, pp. 4832-4837, 2010.

[40] K. J. M. Stibbe, C. Verveer, J. Francke et al., "Comparison of noninvasive assessment to diagnose liver fibrosis in chronic hepatitis B and C patients," Scandinavian Journal of Gastroenterology, vol. 46, no. 7-8, pp. 962-972, 2011.

[41] P. M. Trembling, P. Lampertico, J. Parkes et al., "Performance of Enhanced Liver Fibrosis test and comparison with transient elastography in the identification of liver fibrosis in patients with chronic hepatitis B infection," Journal of Viral Hepatitis, vol. 21, no. 6, pp. 430-438, 2014.

[42] M. Viganò, S. Paggi, P. Lampertico et al., "Dual cut-off transient elastography to assess liver fibrosis in chronic hepatitis B: A cohort study with internal validation," Alimentary Pharmacology \& Therapeutics, vol. 34, no. 3, pp. 353-362, 2011.

[43] X. Cao, Y. Guan, and W. Yu, "Diagnostic study of Fibroscan for liver fibrosis in patients with chronic hepatitis B," Journal of Tropical Medicine, pp. 14-779, 2014.

[44] J. Jia, J. Hou, H. Ding et al., “Transient elastography compared to serum markers to predict liver fibrosis in a cohort of Chinese patients with chronic hepatitis B," Journal of Gastroenterology and Hepatology, vol. 30, no. 4, pp. 756-762, 2015.

[45] S. U. Kim, J. K. Kim, J. Y. Park et al., "Variability in liver stiffness values from different intercostal spaces," Liver International, vol. 29, no. 5, pp. 760-766, 2009.

[46] G. L.-H. Wong, H. L.-Y. Chan, P. C.-L. Choi et al., "Non-invasive algorithm of enhanced liver fibrosis and liver stiffness measurement with transient elastography for advanced liver fibrosis in chronic hepatitis B," Alimentary Pharmacology \& Therapeutics, vol. 39, no. 2, pp. 197-208, 2014.

[47] G. L.-H. Wong, V. W.-S. Wong, P. C.-L. Choi et al., "Metabolic syndrome increases the risk of liver cirrhosis in chronic hepatitis B," Gut, vol. 58, no. 1, pp. 111-117, 2009.

[48] C. R. A. Lesmana, S. Salim, I. Hasan et al., "Diagnostic accuracy of transient elastography (FibroScan) versus the aspartate transaminase to platelet ratio index in assessing liver fibrosis in chronic hepatitis B: the role in primary care setting," Journal of Clinical Pathology, vol. 64, no. 10, pp. 916-920, 2011.

[49] A.-C. Cardoso, R. J. Carvalho-Filho, C. Stern et al., "Direct comparison of diagnostic performance of transient elastography in patients with chronic hepatitis B and chronic hepatitis C," Liver International, vol. 32, no. 4, pp. 612-621, 2012.

[50] X.-B. Chen, X. Zhu, L.-Y. Chen, E.-Q. Chen, and H. Tang, "Accuracy of FibroScan for the diagnosis of liver fibrosis influenced by serum alanine aminotransferase levels in patients with chronic hepatitis B," Chinese Journal of Hepatology, vol. 19, no. 4, pp. 286-290, 2011.

[51] P. Marcellin, M. Ziol, P. Bedossa et al., "Non-invasive assessment of liver fibrosis by stiffness measurement in patients with chronic hepatitis B," Liver International, vol. 29, no. 2, pp. 242247, 2009.
[52] F. Meng, Y. Zheng, Q. Zhang et al., "Noninvasive evaluation of liver fibrosis using real-time tissue elastography and transient elastography (FibroScan)," Journal of Ultrasound in Medicine, vol. 34, no. 3, pp. 403-410, 2015.

[53] K. Osakabe, N. Ichino, T. Nishikawa et al., "Reduction of liver stiffness by antiviral therapy in chronic hepatitis B," Journal of Gastroenterology, vol. 46, no. 11, pp. 1324-1334, 2011.

[54] X. Zhu, L.-C. Wang, E.-Q. Chen et al., "Prospective evaluation of fibroscan for the diagnosis of hepatic fibrosis compared with liver biopsy/AST platelet ratio index and FIB-4 in patients with chronic HBV infection," Digestive Diseases and Sciences, vol. 56, no. 9, pp. 2742-2749, 2011.

[55] H. J. Cho, Y. S. Seo, K. G. Lee et al., "Serum aminotransferase levels instead of etiology affects the accuracy of transient elastography in chronic viral hepatitis patients," Journal of Gastroenterology and Hepatology, vol. 26, no. 3, pp. 492-500, 2011.

[56] L. Castéra, P.-H. Bernard, B. Le Bail et al., "Transient elastography and biomarkers for liver fibrosis assessment and followup of inactive hepatitis B carriers," Alimentary Pharmacology \& Therapeutics, vol. 33, no. 4, pp. 455-465, 2011.

[57] F. Degos, P. Perez, B. Roche et al., "Diagnostic accuracy of FibroScan and comparison to liver fibrosis biomarkers in chronic viral hepatitis: a multicenter prospective study (the FIBROSTIC Study)," Journal of Hepatology, vol. 53, no. 6, pp. 1013-1021, 2010.

[58] J. Cheng, J. Hou, H. Ding et al., "Validation of ten noninvasive diagnostic models for prediction of liver fibrosis in patients with chronic hepatitis B," PLoS ONE, vol. 10, no. 12, Article ID e0144425, 2015.

[59] R. Goyal, S. R. Mallick, M. Mahanta et al., "Fibroscan can avoid liver biopsy in Indian patients with chronic hepatitis B," Journal of Gastroenterology and Hepatology, vol. 28, no. 11, pp. 17381745, 2013.

[60] European Association for the Study of the Liver, "EASL Clinical Practice Guidelines: management of hepatitis C virus infection," Journal of Hepatology, vol. 60, no. 2, pp. 392-420, 2014.

[61] X. Qi, F. Liu, Z. Li et al., "Insufficient accuracy of computed tomography-based portal pressure assessment in hepatitis B virus-related cirrhosis: An analysis of data from CHESS-1601 trial," Journal of Hepatology, vol. 68, no. 1, pp. 210-211, 2017.

[62] Y. Li, Y.-S. Huang, Z.-Z. Wang et al., "Systematic review with meta-analysis: The diagnostic accuracy of transient elastography for the staging of liver fibrosis in patients with chronic hepatitis B," Alimentary Pharmacology \& Therapeutics, vol. 43, no. 4, pp. 458-469, 2016. 


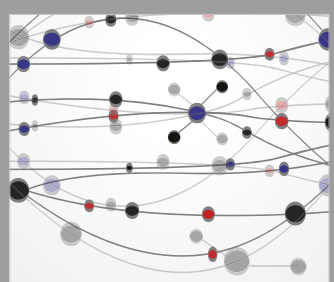

The Scientific World Journal
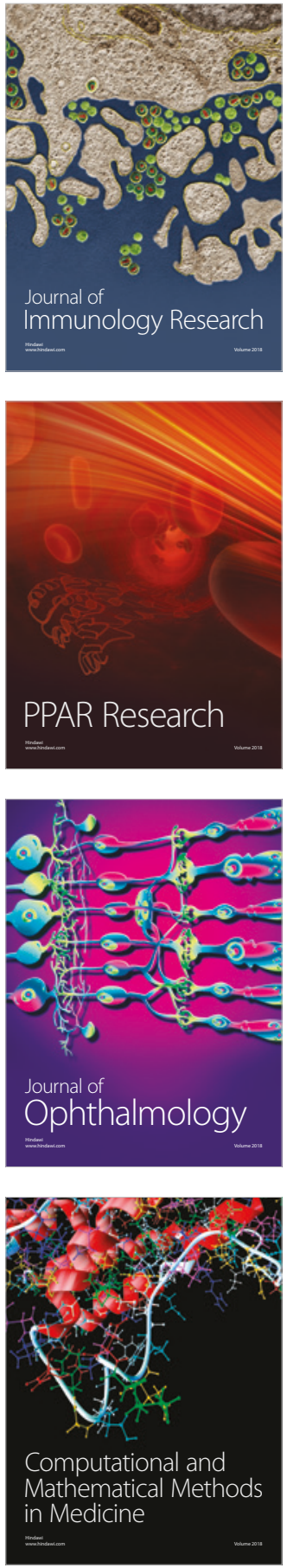

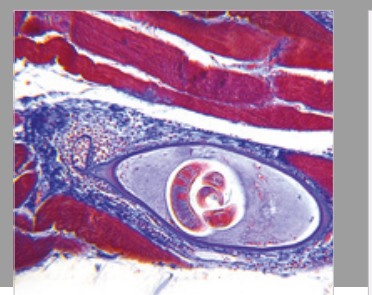

Gastroenterology Research and Practice

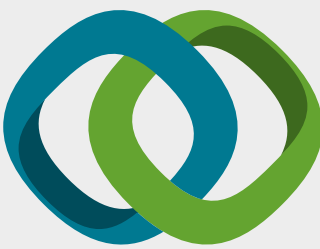

\section{Hindawi}

Submit your manuscripts at

www.hindawi.com
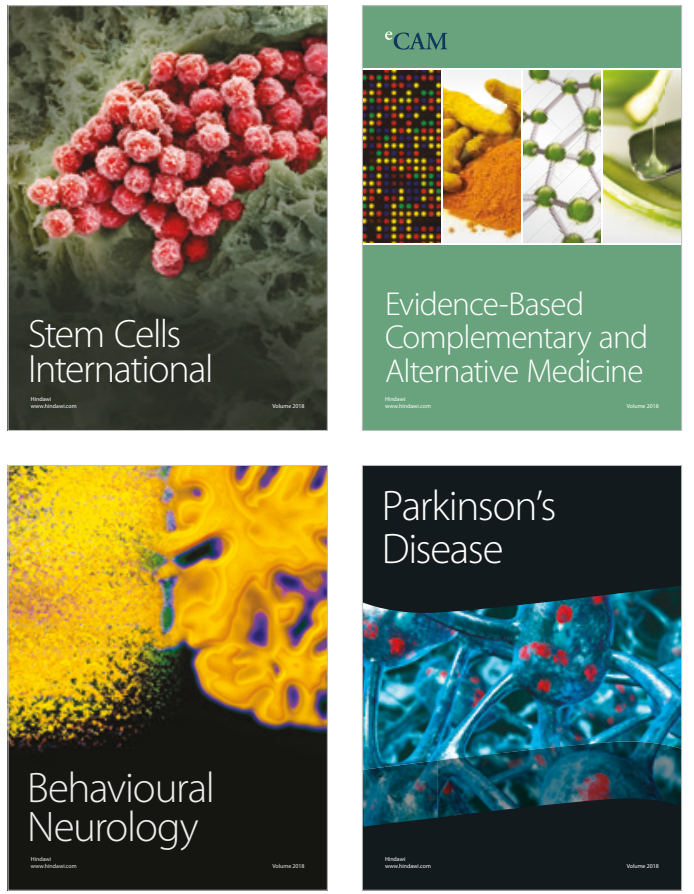

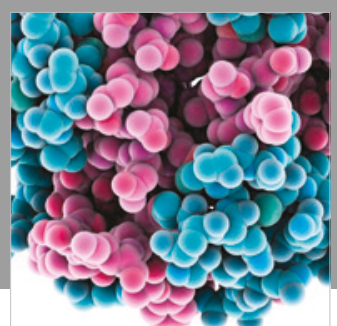

ournal of

Diabetes Research

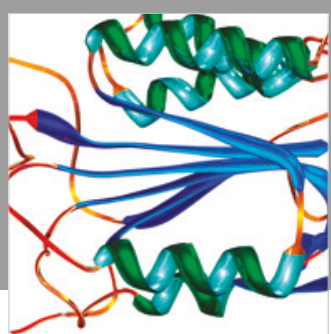

Disease Markers
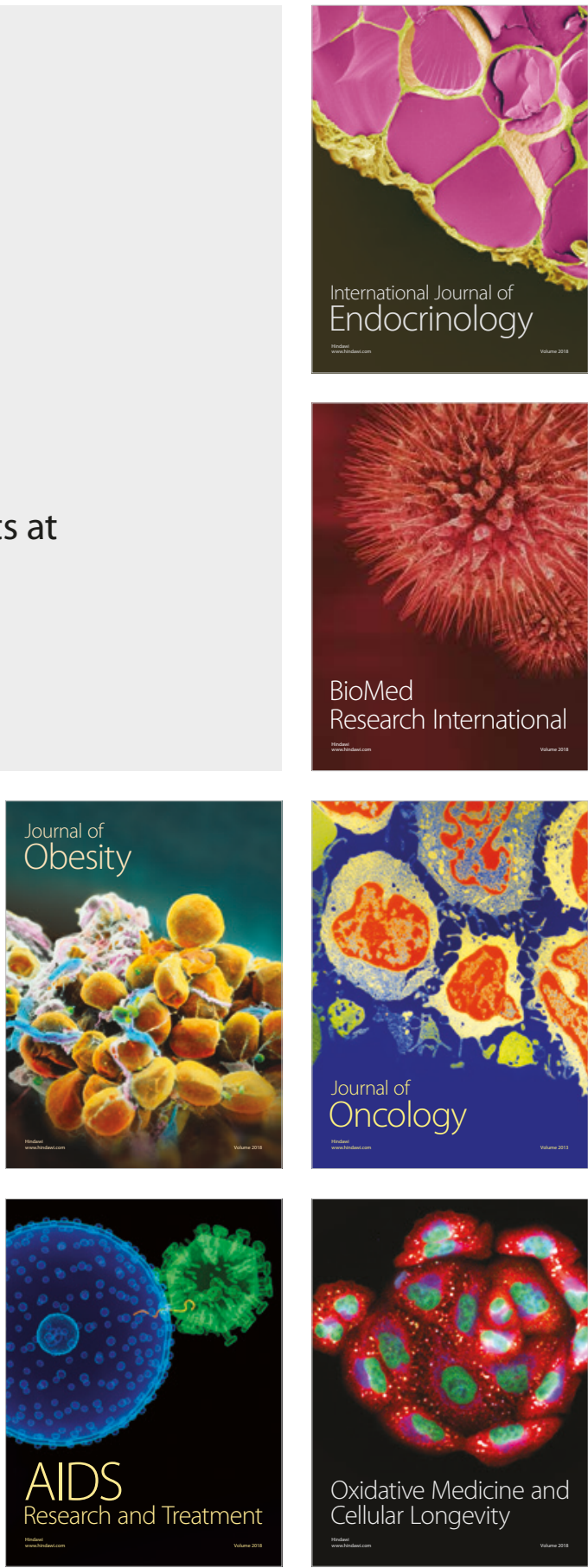